OPEN ACCESS

Edited by:

Ke Nie,

Rutgers Cancer Institute of New Jersey, United States

Reviewed by: Yibao Zhang,

Peking University Cancer Hospital,

China

Yulin Song,

Memorial Sloan Kettering Cancer

Center, United States

*Correspondence: Huidong Wang

wang_hd2010@jlu.edu.cn

Lihua Dong

dlh@jlu.edu.cn

Specialty section: This article was submitted to

Radiation Oncology,

a section of the journal

Frontiers in Oncology

Received: 23 November 2021 Accepted: 17 January 2022

Published: 08 February 2022

Citation:

Sun W, Shi Y, LiY, Ge C, Yang $X$, Xia W, Chen $K$, Wang $L$,

Dong $L$ and Wang $H$ (2022)

Selection Strategy of Jaw Tracking in

VMAT Planning for Lung SBRT.

Front. Oncol. 12:820632.

doi: 10.3389/fonc.2022.820632

\section{Selection Strategy of Jaw Tracking in VMAT Planning for Lung SBRT}

\author{
Wuji Sun ${ }^{1}$, Yinghua Shi ${ }^{1}, Y u L_{i}{ }^{1}$, Chao $\mathrm{Ge}^{1}$, Xu Yang ${ }^{1}$, Wenming Xia ${ }^{1}$, Kunzhi Chen ${ }^{1}$, \\ Libo Wang ${ }^{1}$, Lihua Dong ${ }^{1,2,3^{*}}$ and Huidong Wang ${ }^{1,2^{*}}$ \\ ${ }^{1}$ Department of Radiation Oncology and Therapy, The First Hospital of Jilin University, Changchun, China, ${ }^{2}$ Jilin Provincial \\ Key Laboratory of Radiation Oncology and Therapy, Department of Radiation Oncology and Therapy, The First Hospital of \\ Jilin University, Changchun, China, ${ }^{3}$ National Health Commission (NHC) Key Laboratory of Radiobiology, School of Public \\ Health, Jilin University, Changchun, China
}

Purpose: This study aimed to investigate the dosimetric effect and delivery reliability of jaw tracking (JT) with increasing planning target volume (PTV) for lung stereotactic body radiation therapy (SBRT) plans. A threshold of PTV was proposed as a selection criterion between JT and fixed-jaw (FJ) techniques.

Methods: A total of 28 patients with early-stage non-small-cell lung cancer were retrospectively included. The PTVs ranged from $4.88 \mathrm{cc}$ to $68.74 \mathrm{cc}$, prescribed with $48 \mathrm{~Gy}$ in four fractions. Three-partial-arc volumetric modulated arc therapy (VMAT) plans with FJ and with JT were created for each patient with the same optimization objectives. These two sets of plans were compared using metrics, including conformity index (Cl), $V_{50 \%}, R_{50 \%}, D_{2 \mathrm{~cm}}$, dose-volume parameters of organs at risk, and monitor units (MUs). The ratio of small subfields ( $<3 \mathrm{~cm}$ in either dimension), \%SS, was acquired as a surrogate for the small-field uncertainty. Statistical analyses were performed to evaluate the correlation between the differences in these parameters and the PTV.

Results: The $V_{50 \%}, R_{50 \%}, D_{2 c m}$, and $V_{20 G y}, D_{1,500 c c}$, and $D_{1,000 c c}$ of the lung showed a statistically significant improvement in JT plans as opposed to FJ plans, while the number of MU in JT plans was higher by an average of 1.9\%. Between FJ and JT plans, the PTV was strongly correlated with the differences in $V_{50 \%}$, moderately correlated with those in $V_{20 G y}$ of the lung, and weakly correlated with those in $D_{2 c m}$ and $D_{1,500 c c}$ of the lung. By using JT, \%SS was found to be negatively correlated with the PTV, and the PTV should be at least approximately $12.5 \mathrm{cc}$ for an expected \%SS <50\%, which was 15 cc for a \%SS $<20 \%$ and $20 \mathrm{cc}$ for a $\%$ SS $<5 \%$.

Conclusions: Considering the dosimetric differences and small-field uncertainties, JT could be selected using a PTV threshold, such as 12.5, 15, or $20 \mathrm{cc}$, on the basis of the demand of delivery reliability for lung SBRT.

Keywords: stereotactic body radiation therapy, jaw tracking technique, volumetric modulated arc therapy, planning target volume, small field 


\section{INTRODUCTION}

Stereotactic body radiation therapy (SBRT) has been widely used as an alternative modality to surgery for the treatment of earlystage non-small-cell lung cancer (NSCLC) in recent years (1-3). SBRT features a high dose delivered in few fractions, and a rapid dose fall-off away from the target and optimal target dose conformity are crucial to minimize the normal tissue toxicity (4-8). SBRT demands a high level of delivery accuracy during the treatment, which can be affected by multiple factors, such as beam modeling and machine commissioning $(8,9)$.

With the extensive implementation of SBRT, interest in the use of small photon fields has been rapidly growing (9-11). However, due to lack of lateral charged-particle equilibrium, collimator effects, and limited choices of radiation detectors, small-field dosimetry has always been challenging, and large uncertainties are generally expected in small-field measurements (12-14). Therefore, small-field dosimetry is one of the main factors related to the delivery accuracy of SBRT. In the commissioning of treatment planning systems (TPSs), such as the Eclipse TPS, the beam data for small fields, i.e., below $3 \times 3 \mathrm{~cm}^{2}$, are mostly not included in the beam modeling, except for the carefully measured output factors, which could be used for jaw-defined fields (15).

Volumetric modulated arc therapy (VMAT), as an advanced radiation delivery technique involving simultaneous modulation of the multi-leaf collimator (MLC), dose rate, and gantry speed, could deliver highly conformed doses to the target while minimizing the dose to normal tissues, hence commonly used in SBRT $(16,17)$. Over the past few years, the jaw tracking (JT) technique has been broadly used for VMAT planning to reduce inter-leaf leakage and intra-leaf transmission through MLC (18, 19). Several studies demonstrated that using JT rather than fixedjaw (FJ) technique could lead to improved organ-at-risk (OAR) sparing and superior dose fall-off away from the target (19-22).

The JT technique features continuous adjustment of jaw positions to fit the MLC apertures throughout the treatment process. In smallfield planning, especially for SBRT, the use of JT could result in a large number of subfields below $3 \times 3 \mathrm{~cm}^{2}$, which may cause large discrepancies between calculated and delivered doses (23-25). In the current clinical practice, the FJ technique is generally recommended for small-field planning to achieve minimal calculation uncertainties $(26,27)$. However, the decision-making between JT and FJ is usually based on users' experiences, and a clear consensus is yet to be established. Besides, the correlation between the PTV and benefits from using JT is worth investigating.

This study aimed to investigate the dosimetric advantages and the ratio of small subfields while implementing JT in VMAT planning for lung SBRT with a wide range of tumor sizes and discuss the feasibility of using PTV as a selection criterion between JT and FJ techniques.

\section{MATERIALS AND METHODS}

\section{Patient Immobilization and Simulation}

A total of 28 patients with early-stage peripheral NSCLC treated at the First Hospital of Jilin University between 2017 and 2021 were retrospectively included. The distances between the PTV and the chest wall were $<0.5 \mathrm{~cm}$ for 12 patients, $0.5-1.0 \mathrm{~cm}$ for 6 patients, $1.0-1.5 \mathrm{~cm}$ for 6 patients, and $>1.5 \mathrm{~cm}$ for 4 patients. Patients were immobilized with custom-made negative-pressure vacuum cushions in supine position. Four-dimensional computed tomography (4D-CT) scan was acquired on a Philips Brilliance Big Bore CT scanner with a bellows system (Philips Healthcare, Cleveland, OH, USA). The 4D-CT datasets were reconstructed as 10 respiratory phases with $1.5-\mathrm{mm}$ slice thickness and then transferred to the Varian Eclipse 15.6 TPS.

\section{Target Definition}

Maximum intensity projection (MIP) and average intensity projection (AIP) images were reconstructed in Eclipse. The internal target volume (ITV) was contoured on the MIP and reviewed on each phase of the 4D-CT dataset. The PTV was defined by adding an isotropic $5-\mathrm{mm}$ margin to the ITV. The OARs were contoured on the AIP, including the lung, spinal cord, esophagus, and trachea.

\section{Treatment Planning}

In accordance with the Radiation Therapy Oncology Group (RTOG) 0915 criteria $(6,7)$, a total dose of $48 \mathrm{~Gy}$ in four fractions was prescribed for all patients. Two VMAT-SBRT plans with three partial $\operatorname{arcs}\left(210^{\circ}\right)$ were generated on the AIP for each patient, that is, one with fixed jaws (FJ plan) and the other with jaw tracking (JT plan). The collimator angles were set to $10^{\circ}, 350^{\circ}$, and $80^{\circ}$ for the three arcs of all plans. All plans were optimized using the same objectives and constraints. An upper monitor unit (MU) constraint of 3,500 MU and a moderate Aperture Shape Controller (ASC) setting were used to limit the plan complexity. It should be noted that the jaw-defined fields for FJ plans were all above $3 \times 3 \mathrm{~cm}^{2}$. Plan optimization was performed in Eclipse version 15.6 TPS for a Varian VitalBeam linear accelerator equipped with a Millennium 120 MLC (Varian Medical Systems, Palo Alto, CA) using 6 MV FFF beam. The MLC transmission factor and the dosimetric leaf gap for $6 \mathrm{MV}$ FFF were $1.17 \%$ and $0.14 \mathrm{~cm}$, respectively. The maximum dose rate was $1,400 \mathrm{MU} / \mathrm{min}$. The AcurosXB algorithm was used to calculate the dose distributions with a grid spacing of $1.25 \mathrm{~mm}$. All plans were normalized to achieve the same target coverage, i.e., $95 \%$ of the PTV received $100 \%$ prescription dose.

\section{Plan Evaluation}

The dose-volume parameters of FJ and JT plans were compared, including the following metrics per RTOG 0915 recommendations $(6,7)$ : conformity index $(\mathrm{CI})$, defined as the ratio of prescription isodose volume $\left(\mathrm{V}_{100 \%}\right)$ to the PTV; ratio of $50 \%$ prescription isodose volume to PTV, $\mathrm{R}_{50 \%}$; maximum dose 2 $\mathrm{cm}$ away from the PTV $\left(\mathrm{D}_{2 \mathrm{~cm}}\right.$, in percentage of the prescription dose); percentage of lung receiving at least $20 \mathrm{~Gy}\left(\mathrm{~V}_{20 \mathrm{~Gy}}\right)$; doses to $1,500 \mathrm{cc}\left(\mathrm{D}_{1,500 \mathrm{cc}}\right)$ and $1,000 \mathrm{cc}\left(\mathrm{D}_{1,000 \mathrm{cc}}\right)$ of normal lung; and near-maximum doses to the spinal cord $\left(\mathrm{D}_{0.35 \mathrm{cc}}\right)$, esophagus $\left(D_{5 c c}\right)$, and trachea $\left(D_{4 c c}\right)$. Besides, considering the uncertainty in the small-field dosimetry, the percentage of small subfields $(<3$ $\mathrm{cm}$ in either dimension), \%SS, was calculated for each JT plan. The correlation between \%SS and the PTV was analyzed. 


\section{Data Analysis and Statistics}

Wilcoxon signed-rank tests were used for the comparison between FJ and JT plans. Correlations between the PTV and differences in plan metrics between FJ and JT plans and those between the PTV and \%SS were examined using Spearman's rank correlation coefficient $\left(r_{\mathrm{s}}\right) \cdot\left|r_{\mathrm{s}}\right| \geq 0.7$ was considered a strong correlation, $0.7>\left|r_{\mathrm{s}}\right| \geq 0.5$ was considered a moderate correlation, $0.5>\left|r_{\mathrm{s}}\right| \geq 0.3$ was considered a weak correlation, and $\left|r_{\mathrm{s}}\right|<0.3$ was considered no correlation. A two-tailed $p<0.05$ was considered statistically significant. All statistical analyses were performed using IBM SPSS Statistics 26.0 software (IBM Corporation, Armonk, NY, USA).

\section{RESULTS}

All plans complied with the RTOG 0915 protocol $(6,7)$. The comparison between the dosimetric parameters of JT and FJ plans is summarized in Table 1. $\mathrm{V}_{50 \%}, \mathrm{R}_{50 \%}, \mathrm{D}_{2 \mathrm{~cm}}$, and all parameters of the lung showed a statistically significant improvement in JT plans as opposed to FJ plans, while the number of MU in JT plans was higher by an average of $1.9 \%$. No significant difference was found for $\mathrm{V}_{100 \%}, \mathrm{CI}$, and the parameters of the spinal cord, esophagus, and trachea. The dosimetric distributions of a random patient with a PTV of $16.01 \mathrm{cc}$ are shown in Figure 1. The purple-colored structure indicates the $50 \%$ prescription isodose volume of the JT plan, and the subfigure in the left panel shows the difference in the 50\% prescription isodose volume between JT and FJ plans.

Figure 2 shows the correlations between the PTV and the differences in dosimetric parameters of FJ and JT plans that achieved statistical significance. As indicated in Figure 1, the PTV was strongly correlated with the difference in $\mathrm{V}_{50 \%}$ of the PTV, moderately correlated with that in $\mathrm{V}_{20 \mathrm{~Gy}}$ of the lung, and weakly correlated with those in $D_{2 \mathrm{~cm}}$ and $\mathrm{D}_{1,500 \mathrm{cc}}$ of the lung.

Figure 3 shows the correlation between \%SS and the PTV, including the corresponding curve fitting results, yielding a coefficient of determination $\left(\mathrm{R}^{2}\right)$ of 0.927 . The expected \%SS values are basically $100 \%$ and $0 \%$ for PTVs below $6 \mathrm{cc}$ and above $35 \mathrm{cc}$, respectively, and a near-linear correlation could be seen from 6 to $20 \mathrm{cc}$.

Figure 4 displays the difference of jaw-defined subfield sizes at several gantry angles between JT and FJ plans of a random patient with a PTV of $16.01 \mathrm{cc}$. The average subfield size was reduced to approximately $45 \%$ with the use of JT technique for this patient.

\section{DISCUSSION}

This study investigated the differences in dosimetry and delivery accuracy between JT and FJ plans. One selection strategy by using PTV as a criterion was proposed to choose between JT and FJ techniques for lung SBRT.

A high-dose fall-off is vital to ensure an optimal normal tissue sparing in SBRT. Several studies have proposed that the JT technique could reduce the doses to OARs adjacent to the target with the reduction in the leakage and transmission of the MLC, potentially improving the dose fall-off away from the target $(18,22,27)$. In the present study, Table 1 and Figure 1 show that the dosimetric improvement with the use of JT was in agreement with previous studies (19-22). Moreover, the PTV was found to be related to the magnitude of the improvements, which, to the authors' knowledge, has not been investigated.

Compared with FJ plans, significant improvements were found for $V_{50 \%}, R_{50 \%}, D_{2 c m}$, and $V_{20 G y}, D_{1,500 c c}$, and $D_{1,000 c c}$ of the lung in JT plans. These parameters were all associated with relatively lower dose levels, implying that the JT technique is primarily helpful for the protection of parallel organs where lowdose-volume parameters are more important, such as lung. As the JT technique is characterized by the reduction in leakage and intra-leaf transmission in the MLC, the low-dose region could be somewhat more directly affected, whereas the high-dose region mainly located at the center of the radiation field may be less sensitive. As indicated in Table 1, the $\mathrm{V}_{100 \%}$, CI, and nearmaximum dose parameters of the spinal cord, esophagus, and

TABLE 1 | Comparison between jaw tracking (JT) and fixed-jaw (FJ) plans. The data are shown in mean value \pm standard deviation, along with the Wilcoxon signedrank test $p$-values.

\begin{tabular}{|c|c|c|c|c|}
\hline & Parameter & JT plan & FJ plan & $p$-value \\
\hline \multirow[t]{5}{*}{ PTV } & $V_{100 \%}(c c)$ & $21.02 \pm 15.28$ & $21.03 \pm 15.31$ & 0.666 \\
\hline & $\mathrm{V}_{50 \%}(\mathrm{cc})$ & $80.59 \pm 50.56$ & $82.19 \pm 51.93$ & $<0.001^{\star}$ \\
\hline & $\mathrm{Cl}$ & $0.99 \pm 0.02$ & $0.99 \pm 0.02$ & 0.909 \\
\hline & $\mathrm{R}_{50 \%}$ & $4.00 \pm 0.47$ & $4.07 \pm 0.47$ & $<0.001^{\star}$ \\
\hline & $\mathrm{D}_{2 \mathrm{~cm}}(\mathrm{cGy})$ & $2,018.22 \pm 209.72$ & $2,035.51 \pm 213.48$ & $0.006^{*}$ \\
\hline \multirow[t]{3}{*}{ Lung } & $\mathrm{V}_{20 G y}(\%)$ & $2.82 \pm 1.67$ & $2.85 \pm 1.69$ & $0.001^{*}$ \\
\hline & $\mathrm{D}_{1,500 c c}$ (cGy) & $34.77 \pm 26.04$ & $35.21 \pm 26.55$ & $<0.001^{\star}$ \\
\hline & $\mathrm{D}_{1,000 \mathrm{cc}}$ (cGy) & $88.42 \pm 60.99$ & $89.44 \pm 61.62$ & $0.001^{*}$ \\
\hline Spinal cord & $\mathrm{D}_{0.35 \mathrm{cc}}$ (cGy) & $683.59 \pm 296.19$ & $671.16 \pm 287.52$ & 0.285 \\
\hline Esophagus & $D_{5 c c}(c G y)$ & $357.7 \pm 243.02$ & $358.8 \pm 242.57$ & 0.472 \\
\hline Trachea & $\mathrm{D}_{4 \mathrm{cc}}$ (cGy) & $817.41 \pm 516.26$ & $806.8 \pm 498.88$ & 0.177 \\
\hline Monitor unit & $\mathrm{MU}$ & $3,701.98 \pm 217.39$ & $3,633.75 \pm 236.70$ & $0.001^{*}$ \\
\hline
\end{tabular}

*Statistically significant $(p<0.05)$.

PTV, planning target volume. 


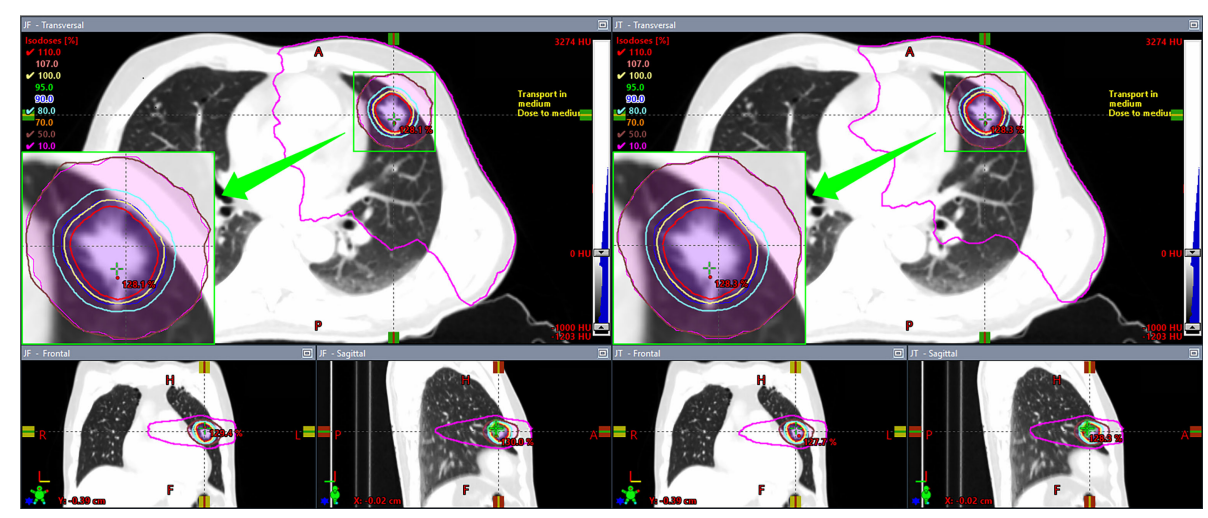

FIGURE 1 | Comparison of dosimetric distributions between fixed-jaw (FJ; left panel) and jaw tracking (JT; right panel) plans of a patient.
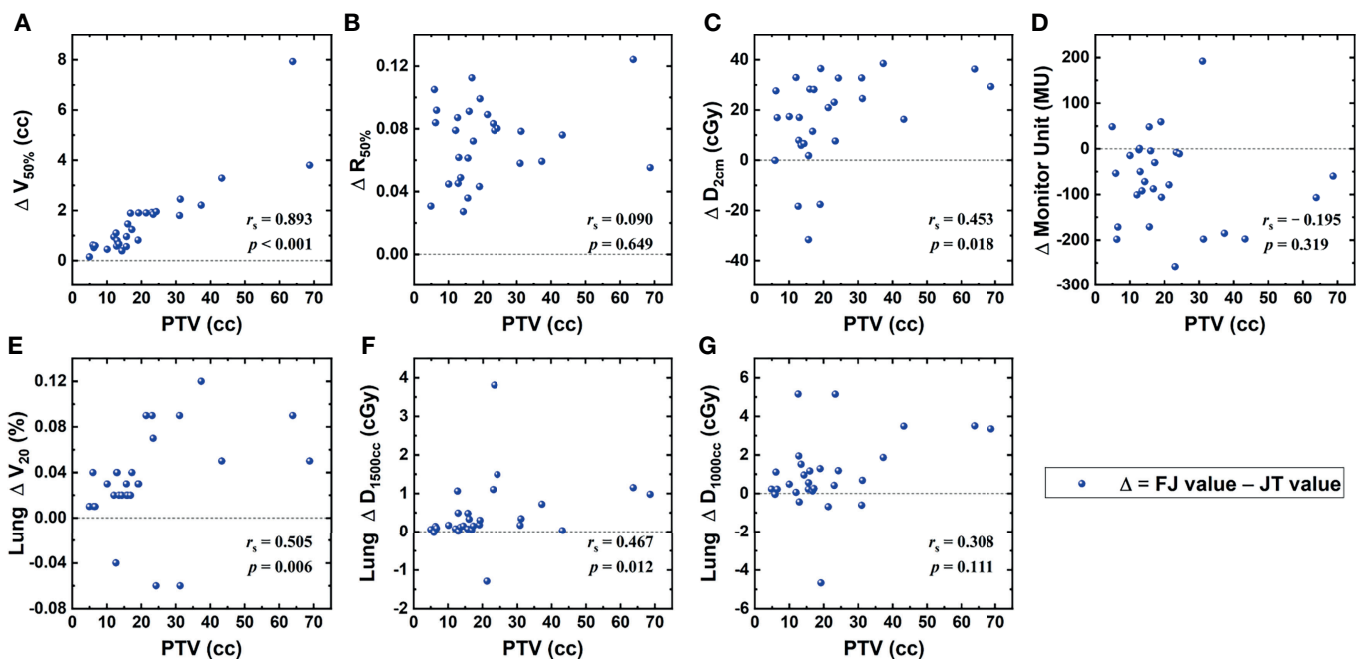

FIGURE 2 | Correlations between the planning target volume (PTV) and the differences in dosimetric parameters between fixed-jaw (FJ) and jaw tracking (JT) plans, including $V_{50 \%}$ of the PTV (A), $R_{50 \%}(\mathbf{B}), D_{2 c m}(\mathbf{C})$, monitor unit (MU) (D), and $V_{20 G y}(\mathbf{E}), D_{1,500 c c}(\mathbf{F})$, and $D_{1,000 c c}(\mathbf{G})$ of the lung.

trachea showed no significant differences between FJ and JT plans. In addition, the number of MU in JT plans were higher by an average of $1.9 \%$, implying a small increase in the plan complexity. Among the parameters where significant improvements were found in JT plans, the magnitudes of the improvements in $\mathrm{V}_{50 \%}, \mathrm{D}_{2 \mathrm{~cm}}$, and $\mathrm{V}_{20 \mathrm{~Gy}}$ and $\mathrm{D}_{1,500 \mathrm{cc}}$ of the lung showed positive correlations with the PTV, as indicated in Figure 1. For smaller PTVs, the dosimetric differences between JT and FJ may be relatively indistinguishable, and the choice between JT and FJ should be primarily based upon other factors, such as delivery accuracy.

A high level of delivery accuracy is crucial for the implementation of SBRT. Due to the extensive use of smallfield dose delivery in SBRT and the difficulties in measurements of small field, a number of reviews, guidelines, and recommendations have been published on the subject of small- field dosimetry, such as the IPEM report 103 (12), the IAEAAAPM TRS-483 code of practice (13), and the more recent AAPM TG-155 report (14). However, due to limited resources or lack of qualified staff, many treatment centers may not be able to perform reliable small-field measurements, and the avoidance of uncertainties would be practical. For the Eclipse TPS, the smallfield beam data will not affect the calculation results for the MLCdefined field where MLC is below jaws in the linac because the backscatter is determined from the jaw-defined field (15). When small jaw-defined fields are used, the measured output factor may affect the calculation accuracy for small-field cases (15). In JT plans with a small PTV, potential uncertainties could be brought by small subfield calculations (23-25). Therefore, it has been suggested that the FJ technique should be used in smallfield planning to achieve minimal calculation uncertainties $(26,27)$. 


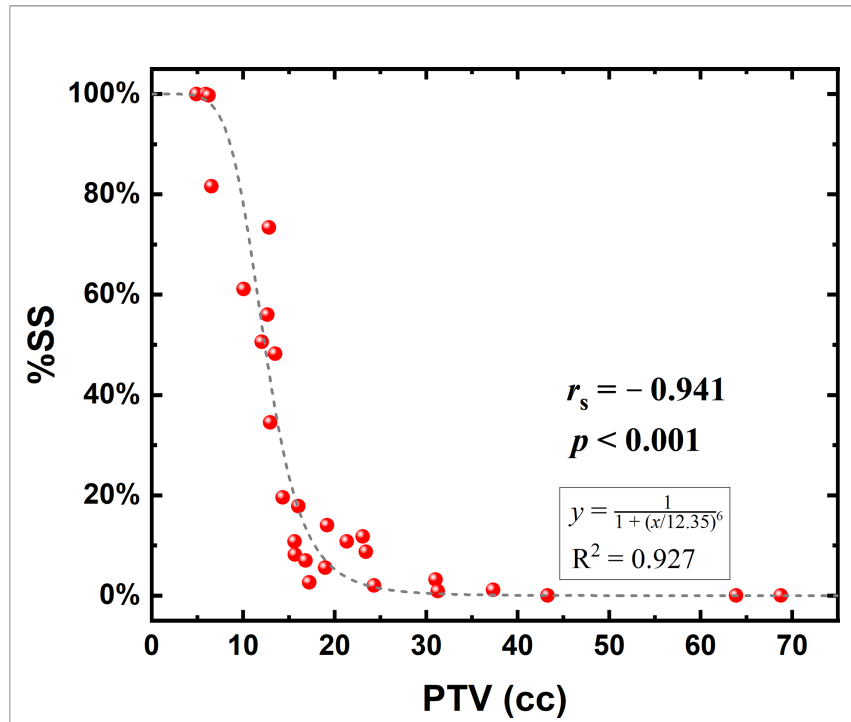

FIGURE 3 | Correlation between the planning target volume (PTV) and the percentage of small subfields (\%SS) in jaw tracking (JT) plans.

In principle, to assess the difference in delivery accuracy between FJ and JT plans, measurement-based dose verification should be performed. Small-field uncertainty is one of the main contributors to the discrepancy between calculated and delivered doses. In accordance with the corresponding guidelines and recommendations $(12-14,28)$, the delivered dose for several FJ and JT plans was measured in the present study. However, due to the large uncertainties in the small-field data acquisition, TPS commissioning, and dose verification, obtaining reliable results to demonstrate the differences in the delivery accuracy between FJ and JT plans was difficult. Besides, discrepancies are inevitable in the TPS commissioning among linacs and treatment centers, and such could further undermine the validity of measurements. Therefore, the quantity \%SS was used as a surrogate for the small-field uncertainty, and a higher \%SS value indicates a potentially lower delivery reliability.
Figure 3 shows that while using JT in VMAT planning, the variation of \%SS occurred primarily in the PTV range of 6-20 cc, where a near-linear decrease was found with increasing PTV. As indicated above, the benefit of JT for small PTV is relatively trivial, and the delivery accuracy is a more important factor to be considered. Therefore, a threshold of PTV could be used as a decisive criterion for the use of JT. For example, the PTV should be at least approximately $12.5 \mathrm{cc}$ for an expected $\% \mathrm{SS}<50 \%$, at least $15 \mathrm{cc}$ for an expected \%SS $<20 \%$, at least $18 \mathrm{cc}$ for an expected $\% \mathrm{SS}<10 \%$, and at least $20 \mathrm{cc}$ for an expected $\% \mathrm{SS}<5 \%$. With a PTV higher than $35 \mathrm{cc}$, the occurrence rate of small subfields in VMAT plans could be considered zero.

In addition, the intra-fraction motion of the target may cause a dose deviation between the calculated and delivered dose due to dose blurring and interplay effects between MLC and tumor motion (29-31), especially for a small-sized target $(32,33)$. Besides, the above results could also be affected by other factors, such as the plan complexity and tumor shapes (34-36). In the present study, an MU constraint and moderate ASC were used to achieve a relatively lower plan complexity and potentially reduce the \%SS. Moreover, an irregular tumor with certain volume could lead to a higher \%SS. Thus, this criterion should be applied on the basis of clinical circumstances. Although statistically significant dosimetric benefits were demonstrated in the above results, further clinical investigation is required to verify the outcome.

\section{CONCLUSIONS}

In this study, the correlation between the magnitude of dosimetric improvement of implementing JT and the PTV in VMAT planning for lung SBRT was investigated, and a quantity \%SS was proposed, i.e., the ratio of small subfields in VMAT plans, as a surrogate for the uncertainty in small-dose dosimetry. The PTV was found to be positively correlated with the dosimetric benefits of JT and negatively correlated with the \% SS. By taking the benefits of JT and the small-dose uncertainty

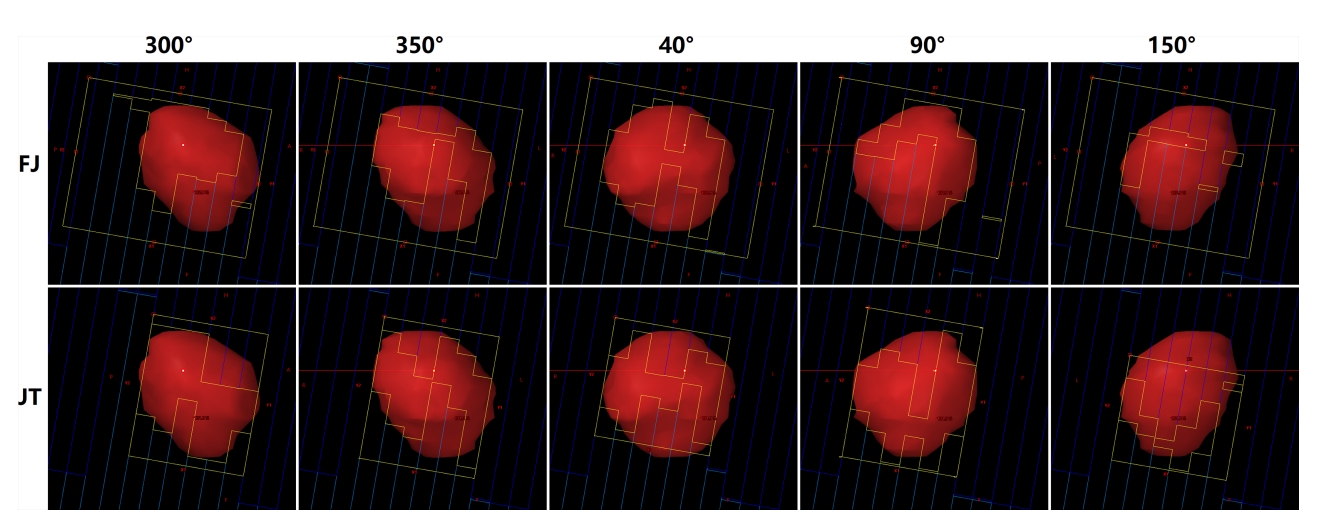

FIGURE 4 | Beam's eye views at five gantry angles in fixed-jaw (FJ; upper panel) and jaw tracking (JT) plans (lower panel) of a patient. 
into account altogether, a selection strategy between JT and FJ was proposed using a PTV threshold.

\section{DATA AVAILABILITY STATEMENT}

The raw data supporting the conclusions of this article will be made available by the authors without undue reservation.

\section{ETHICS STATEMENT}

Written informed consent was not obtained from the individual(s) for the publication of any potentially identifiable images or data included in this article.

\section{REFERENCES}

1. Timmerman RD, Paulus R, Pass HI, Gore EM, Edelman MJ, Galvin J, et al. Stereotactic Body Radiation Therapy for Operable Early-Stage Lung Cancer: Findings From the NRG Oncology RTOG 0618 Trial. JAMA Oncol (2018) 4:1263-6. doi: 10.1001/jamaoncol.2018.1251

2. Fakiris AJ, McGarry RC, Yiannoutsos CT, Papiez L, Williams M, Henderson MA, et al. Stereotactic Body Radiation Therapy for Early-Stage non-SmallCell Lung Carcinoma: Four-Year Results of a Prospective Phase II Study. Int J Radiat Oncol Biol Phys (2009) 75:677-82. doi: 10.1016/j.ijrobp.2008.11.042

3. Nagata Y, Takayama K, Matsuo Y, Norihisa Y, Mizowaki T, Sakamoto T, et al. Clinical Outcomes of a Phase I/II Study of 48 Gy of Stereotactic Body Radiotherapy in 4 Fractions for Primary Lung Cancer Using a Stereotactic Body Frame. Int J Radiat Oncol Biol Phys (2005) 63:1427-31. doi: 10.1016/ j.ijrobp.2005.05.034

4. Ball D, Mai GT, Vinod S, Babington S, Ruben J, Kron T, et al. Stereotactic Ablative Radiotherapy Versus Standard Radiotherapy in Stage 1 non-SmallCell Lung Cancer (TROG 09.02 CHISEL): A Phase 3, Open-Label, Randomised Controlled Trial. Lancet Oncol (2019) 20:494-503. doi: 10.1016/S1470-2045(18)30896-9

5. Bezjak A, Paulus R, Gaspar LE, Timmerman RD, Straube WL, Ryan WF, et al. Safety and Efficacy of a Five-Fraction Stereotactic Body Radiotherapy Schedule for Centrally Located Non-Small-Cell Lung Cancer: NRG Oncology/RTOG 0813 Trial. J Clin Oncol (2019) 37:1316-25. doi: 10.1200/ JCO.18.00622

6. Videtic GM, Paulus R, Singh AK, Chang JY, Parker W, Olivier KR, et al. LongTerm Follow-Up on NRG Oncology RTOG 0915 (NCCTG N0927): A Randomized Phase 2 Study Comparing 2 Stereotactic Body Radiation Therapy Schedules for Medically Inoperable Patients With Stage I Peripheral Non-Small Cell Lung Cancer. Int J Radiat Oncol Biol Phys (2019) 103:1077-84. doi: 10.1016/j.ijrobp.2018.11.051

7. Videtic GM, Hu C, Singh AK, Chang JY, Parker W, Olivier KR, et al. A Randomized Phase 2 Study Comparing 2 Stereotactic Body Radiation Therapy Schedules for Medically Inoperable Patients With Stage I Peripheral Non-Small Cell Lung Cancer: NRG Oncology RTOG 0915 (NCCTG N0927). Int J Radiat Oncol Biol Phys (2015) 93:757-64. doi: 10.1016/j.ijrobp.2015.07.2260

8. Benedict SH, Yenice KM, Followill D, Galvin JM, Hinson W, Kavanagh B, et al. Stereotactic Body Radiation Therapy: The Report of AAPM Task Group 101. Med Phys (2010) 37:4078-101. doi: 10.1118/1.3438081

9. ICRU. ICRU Report 91: Prescribing, Recording, and Reporting of Stereotactic Treatments With Small Photon Beams. J Int Comm Radiat Units Meas (2014) 2);14(2):1-145. doi: 10.1093/jicru_ndx010

10. Alfonso R, Andreo P, Capote R, Huq MS, Kilby W, Kjall P, et al. A New Formalism for Reference Dosimetry of Small and Nonstandard Fields. Med Phys (2008) 35:5179-86. doi: 10.1118/1.3005481

\section{AUTHOR CONTRIBUTIONS}

Conception and design: HW. Acquisition of data: KC, YL, WX, CG, XY, and LW. Analysis and interpretation of data: WS, YS, and HW. Drafting of the article: WS and HW. Critical revision of the article: LD and HW. All authors contributed to the article and approved the submitted version.

\section{FUNDING}

This work was supported by the Jilin Scientific and Technological Development Program (No. 20200601005JC) and the Jilin Province Special Project of Medical and Health Talents (No. JLSCZD2019-032).

11. Das IJ, Ding GX, Ahnesjo A. Small Fields: Nonequilibrium Radiation Dosimetry. Med Phys (2008) 35:206-15. doi: 10.1118/1.2815356

12. IPEM. Small Field MV Photon Dosimetry, IPEM Report No. 103. New York, UK: Institute of Physics and Engineering in Medicine (2010).

13. IAEA. Dosimetry of Small Static Fields Used in External Beam Radiotherapy: An IAEA-AAPM International Code of Practice for Reference and Relative Dose Determination, Technical Report Series No. 483. Vienna, Austria: International Atomic Energy Agency (2017).

14. Das IJ, Francescon P, Moran JM, Ahnesjo A, Aspradakis MM, Cheng CW, et al. Report of AAPM Task Group 155: Megavoltage Photon Beam Dosimetry in Small Fields and non-Equilibrium Conditions. Med Phys (2021) 48:e886921. doi: 10.1002/mp.15030

15. Torsti T, Korhonen L, PetäjäV V. Using Varian Photon Beam Source Model for Dose Calculation of Small Fields. Clinical Perspectives. Helsinki, Finland: Varian Medical Systems (2013).

16. Hernandez V, Hansen CR, Widesott L, Back A, Canters R, Fusella M, et al. What is Plan Quality in Radiotherapy? The Importance of Evaluating Dose Metrics, Complexity, and Robustness of Treatment Plans. Radiother Oncol (2020) 153:26-33. doi: 10.1016/j.radonc.2020.09.038

17. Zhang GG, Ku L, Dilling TJ, Stevens CW, Zhang RR, Li W, et al. Volumetric Modulated Arc Planning for Lung Stereotactic Body Radiotherapy Using Conventional and Unflattened Photon Beams: A Dosimetric Comparison With 3D Technique. Radiat Oncol (2011) 6:152. doi: 10.1186/1748-717X-6-152

18. Feng Z, Wu H, Zhang Y, Zhang Y, Cheng J, Su X. Dosimetric Comparison Between Jaw Tracking and Static Jaw Techniques in Intensity-Modulated Radiotherapy. Radiat Oncol (2015) 10:28. doi: 10.1186/s13014-015-0329-4

19. Wu H, Jiang F, Yue H, Hu Q, Zhang J, Liu Z, et al. A Comparative Study of Identical VMAT Plans With and Without Jaw Tracking Technique. J Appl Clin Med Phys (2016) 17:133-41. doi: 10.1120/jacmp.v17i5.6252

20. Murakami Y, Magome T, Matsubayashi F, Takahashi R, Arima M, Kamima T, et al. Evaluation of Organ-at-Risk Dose Reduction With Jaw Tracking Technique in Flattening Filter-Free Beams in Lung Stereotactic Body Radiation Therapy. Phys Med (2019) 61:70-6. doi: 10.1016/j.ejmp.2019.04.018

21. Xie K, Sun H, Gao L, Lin T, Sui J, Ni X. A Comparative Study of Identical VMAT About Two Adjacent Targets With and Without Fixed-Jaw Technique. Radiat Oncol (2019) 14:75. doi: 10.1186/s13014-019-1284-2

22. Pokhrel D, Sanford L, Halfman M, Molloy J. Potential Reduction of Lung Dose via VMAT With Jaw Tracking in the Treatment of Single-Isocenter/ Two-Lesion Lung SBRT. J Appl Clin Med Phys (2019) 20:55-63. doi: 10.1002/ acm 2.12580

23. Lechner W, Primessnig A, Nenoff L, Wesolowska P, Izewska J, Georg D. The Influence of Errors in Small Field Dosimetry on the Dosimetric Accuracy of Treatment Plans. Acta Oncol (2020) 59:511-7. doi: 10.1080/0284186X. 2019.1685127

24. Wolfs CJA, Swinnen ACC, Nijsten S, Verhaegen F. Should Dose From Small Fields be Limited for Dose Verification Procedures?: Uncertainty Versus Small 
Field Dose in VMAT Treatments. Phys Med Biol (2018) 63:20NT01. doi: 10.1088/1361-6560/aae338

25. Palmans H, Andreo P, Huq MS, Seuntjens J, Christaki KE, Meghzifene A. Dosimetry of Small Static Fields Used in External Photon Beam Radiotherapy: Summary of TRS-483, the IAEA-AAPM International Code of Practice for Reference and Relative Dose Determination. Med Phys (2018) 45:e1123-45. doi: $10.1002 / \mathrm{mp} .13208$

26. Torres Valderrama A, Olaciregui-Ruiz I, Gonzalez P, Perik T, Mijnheer B, Mans A. Portal Dosimetry of Small Unflattened Beams. Phys Med Biol (2021) 66:11NT01. doi: 10.1088/1361-6560/ab843d

27. Swinnen AC, Ollers MC, Roijen E, Nijsten SM, Verhaegen F. Influence of the Jaw Tracking Technique on the Dose Calculation Accuracy of Small Field VMAT Plans. J Appl Clin Med Phys (2017) 18:186-95. doi: 10.1002/ acm2.12029

28. Miften M, Olch A, Mihailidis D, Moran J, Pawlicki T, Molineu A, et al. Tolerance Limits and Methodologies for IMRT Measurement-Based Verification QA: Recommendations of AAPM Task Group No. 218 Med Phys (2018) 45:e53-83. doi: 10.1002/mp.12810

29. Ong CL, Dahele M, Slotman BJ, Verbakel WF. Dosimetric Impact of the Interplay Effect During Stereotactic Lung Radiation Therapy Delivery Using Flattening Filter-Free Beams and Volumetric Modulated Arc Therapy. Int $J$ Radiat Oncol Biol Phys (2013) 86:743-8. doi: 10.1016/j.ijrobp.2013.03.038

30. Sande EPS, Acosta Roa AM, Hellebust TP. Dose Deviations Induced by Respiratory Motion for Radiotherapy of Lung Tumors: Impact of CT Reconstruction, Plan Complexity, and Fraction Size. J Appl Clin Med Phys (2020) 21:68-79. doi: 10.1002/acm2.12847

31. Burton A, Offer K, Hardcastle N. A Robust VMAT Delivery Solution for Single-Fraction Lung SABR Utilizing FFF Beams Minimizing Dosimetric Compromise. J Appl Clin Med Phys (2020) 21:299-304. doi: 10.1002/ acm2.12919

32. Zheng D, Zhang Q, Liang $\mathrm{X}$, Zhu $\mathrm{X}$, Verma $\mathrm{V}$, Wang $\mathrm{S}$, et al. Effect of the Normalized Prescription Isodose Line on the Magnitude of Monte Carlo vs. Pencil Beam Target Dose Differences for Lung Stereotactic Body
Radiotherapy. J Appl Clin Med Phys (2016) 17:48-58. doi: 10.1120/ jacmp.v17i4.5965

33. Edvardsson A, Nordstrom F, Ceberg C, Ceberg S. Motion Induced Interplay Effects for VMAT Radiotherapy. Phys Med Biol (2018) 63:085012. doi: 10.1088/1361-6560/aab957

34. Keall PJ, Mageras GS, Balter JM, Emery RS, Forster KM, Jiang SB, et al. The Management of Respiratory Motion in Radiation Oncology Report of AAPM Task Group 76. Med Phys (2006) 33:3874-900. doi: 10.1118/1.2349696

35. Schwarz M, Cattaneo GM, Marrazzo L. Geometrical and Dosimetrical Uncertainties in Hypofractionated Radiotherapy of the Lung: A Review. Phys Med (2017) 36:126-39. doi: 10.1016/j.ejmp.2017.02.011

36. Hoffman D, Dragojevic I, Hoisak J, Hoopes D, Manger R. Lung Stereotactic Body Radiation Therapy (SBRT) Dose Gradient and PTV Volume: A Retrospective Multi-Center Analysis. Radiat Oncol (2019) 14:162. doi: 10.1186/s13014-019-1334-9

Conflict of Interest: The authors declare that the research was conducted in the absence of any commercial or financial relationships that could be construed as a potential conflict of interest.

Publisher's Note: All claims expressed in this article are solely those of the authors and do not necessarily represent those of their affiliated organizations, or those of the publisher, the editors and the reviewers. Any product that may be evaluated in this article, or claim that may be made by its manufacturer, is not guaranteed or endorsed by the publisher.

Copyright $(2022$ Sun, Shi, Li, Ge, Yang, Xia, Chen, Wang, Dong and Wang. This is an open-access article distributed under the terms of the Creative Commons Attribution License (CC BY). The use, distribution or reproduction in other forums is permitted, provided the original author(s) and the copyright owner(s) are credited and that the original publication in this journal is cited, in accordance with accepted academic practice. No use, distribution or reproduction is permitted which does not comply with these terms. 